\title{
1 Functional characterization of the $A B F$ gene family in upland cotton (Gossypium hirsutum L.)
}

3 Tyson C. C. Kerr ${ }^{1,2}$, Haggag Abdel-Mageed ${ }^{1,3}$, MiYoung Kang ${ }^{1}$, Dakota Cryer ${ }^{1}$, and Randy D. Allen ${ }^{1, *}$

$5{ }^{1}$ Institute for Agricultural Biosciences, Oklahoma State University, Ardmore, OK

$6 \quad{ }^{2}$ Department of Biochemistry and Molecular Biology, Oklahoma State University, Stillwater, OK

7 3. Department of Agricultural Botany, Faculty of Agriculture, Cairo University, Giza 12613, Egypt

10 Tyson C. C. Kerr: tyson.kerr@ okstate.edu

11 Haggag Abdel-Mageed: haggag@okstate.edu

12 MiYoung Kang: miyoung.kang@okstate.edu

13 Dakota Cryer: dcryer@ okstate.edu

14

*Corresponding Author: $\quad$ Randy D. Allen

23 Submission date:

24 Tables and figures: 1 table, 6 figures; figs. 3 and 6 in color in print and online.

25 Word count:

26 Supplementary data: 4 tables, 2 figures. 


\section{Functional characterization of the $A B F$ gene family in upland cotton (Gossypium hirsutum L.)}

34 Running title: Functional characterization of the $A B F s$ from upland cotton

\section{Highlight}

37 The Gossypium hirsutum ABF homeologs are differentially expressed in response to abiotic stress, and 38 their ectopic expression in Arabidopsis can confer increased water deficit tolerance.

\section{Abstract}

41 The AREB/ABF bZIP transcription factors play a pivotal role in abscisic acid-dependent abiotic stress-

42 responsive gene expression. Despite the perennial damage and reduced productivity that result from

43 water-deficit and unpredictable early season temperature fluctuations, these critical genes have not been

44 previously examined in upland cotton (Gossypium hirsutum). Here, we report the isolation of the G.

45 hirsutum $A B F$ homologs, characterization of their expression patterns in response to abiotic stress

46 treatments, and examination of their functions through heterologous ectopic expression in Arabidopsis.

47 As expected for an allotetraploid, G. hirsutum $A B F$ homologs are present in the genome as

48 homeologous pairs. These genes are differentially expressed, both among the homologs and within the

49 homeologous pairs, in response to exogenous abscisic acid (ABA) application, dehydration, and

50 chilling temperatures. Furthermore, heterologous ectopic expression of many of the G. hirsutum ABF

51 genes in Arabidopsis conferred increased tolerance to water deficit and osmotic stress, as well as cold

52 tolerance, in a gene specific manner. These results indicate the G. hirsutum ABF homologs are

53 functional in Arabidopsis and, as in other species, are likely to play an essential role in the abiotic stress 54 response.

56 Key words: abiotic stress, abscisic acid, AREB/ABF, cold tolerance, cotton, drought tolerance, 57 tetraploid

59 Abbreviations: $\mathrm{ABA}, \mathrm{ABF}, \mathrm{ABRE}, \mathrm{AREB}$ 


\section{Introduction}

62 Plants experience damage and reduced productivity as a result of exposure to stressful environmental

63 conditions including water deficit and temperature extremes (Boyer, 1982; Bray et al., 2000; Wang et

64 al., 2003). The pernicious effects of abiotic stress are especially problematic in agricultural settings,

65 where economic viability is dependent on predictable, high yields. Cultivated upland cotton

66 (Gossypium hirsutum L.) is particularly susceptible to these acute effects, as it is grown mainly in arid

67 or semi-arid regions where rainfall is limited and early and late season temperatures can fluctuate

68 widely. These conditions make rain-fed production difficult and risky, therefore, more often than not,

69 irrigation is required to consistently produce profitable yields. With the depletion of groundwater

70 resources, the diversion of surface water to other uses, and increasingly extreme and unpredictable

71 temperature fluctuations, the need to understand the mechanisms used by plants, including cotton, to

72 acclimate to stressful conditions and the development of strategies to optimize these systems in order to

73 produce varieties that can remain productive with less water, has become a priority.

75 Plants have robust abiotic stress responsive networks that include myriad differentially regulated genes

76 (Wang et al., 2003; Bartels and Sunkar, 2005; Yoshida et al., 2014). Among these, the abscisic acid

77 (ABA)-responsive element binding proteins/ABRE-binding factors (AREB/ABFs) have been identified

78 as essential regulators of the osmotic stress response (Yamaguchi-Shinozaki and Shinozaki 2006; Fujita

79 et al., 2013; Yoshida et al., 2015). The expression of many of the members of this small sub-family of

80 transcription factors is induced in response to ABA and various other abiotic stressors, and in turn, they

81 modulate the expression of downstream target genes that ultimately result in the up-regulation of

82 abiotic stress-protective factors including membrane and protein stabilizing molecules, antioxidants,

83 and the accumulation of osmocompatible solutes (Wang et al., 2003; Reddy et al., 2004).

85 As ABA-dependent, bZIP transcription factors, the AREB/ABFs interact with the conserved cis-acting

86 ABA-responsive element (ABRE: PyACGTGG/TC) found in the 5' flanking regions of many ABA

87 responsive genes (Choi et al., 2000; Kang et al., 2002; Fujii et al., 2009; Yoshida et al., 2010; Yoshida

88 et al., 2014). Nine $A R E B / A B F$ family members have been identified in Arabidopsis. Of these, three are

89 induced by osmotic stress: $A R E B 1 / A B F 2, A R E B 2 / A B F 4$, and $A B F 3$; a fourth, $A B F 1$, has been shown to

90 be a functional homolog (Yoshida et al., 2015). These Arabidopsis AREB/ABF paralogs contain the

91 basic region and the leucine repeats characteristic of the bZIP domain, and five conserved Ser/Thr 
92 kinase phosphorylation sites (RXXS/T), that are phosphorylated by SnRK2 protein kinases. Although

93 many of these Arabidopsis $A R E B / A B F$ genes are induced by similar abiotic stressors, and their target

94 genes overlap, each exhibits unique temporal and spatial expression patterns (Choi et al., 2000; Fujita

95 et al., 2005; Fujii et al., 2009; Fujita et al., 2013; Yoshida et al., 2015).

97 Ectopic over-expression of this subset of genes from the AREB/ABF family in Arabidopsis has been

98 shown to confer increased tolerance to various abiotic stressors, as a result of their positive regulation

99 of ABA signaling (Kim et al., 2004; Fujita et al., 2005; Chinnusamy et al., 2006; Novillo et al., 2007;

100 Fujii et al., 2009; Yoshida et al., 2010; Medina et al., 2011). These studies show that these AREB/ABF

101 transcription factors play an essential role in the response to abiotic stress, and thus, have been

102 extensively examined in model species. Although the endogenous ectopic expression of these

103 Arabidopsis AREB/ABF genes confers increased abiotic stress tolerance, these improvements are often 104 also accompanied by slower vegetative growth and delayed reproduction.

105

106 Despite the economic importance of cotton as the world's primary source of natural fiber, accounting

107 for $40 \%$ of all textile fibers produced, the $A B F$ gene family has not been fully characterized in $G$.

108 hirsutum, the most commonly cultivated cotton species (Wendel and Cronn, 2003; Meyer et al., 2007;

109 Osakwe, 2009). This is likely due, at least in part, to the allotetraploid nature of the G. hirsutum genome

110 (Wendel \& Cronn 2003; Chaudhary et al., 2009). Here, we characterize the G. hirsutum AREB/ABF

111 homologs (hereinafter $G h A B F s$ ), including their expression in response to various abiotic stressors and

112 their ability to confer improved abiotic stress tolerance when ectopically expressed in Arabidopsis.

113 Furthermore, to address the putative tradeoff between improved stress tolerance and developmental

114 delay, multiple independent transgenic lines, with various levels of ectopic expression, are evaluated

115 for each $G h A B F$ transgenic gene construct to ascertain if there is an acceptable balance of positive and

116 negative functional effects.

118 Materials and methods

119 Gossypium ABF homolog isolation and phylogenetic analysis

120 Gossypium arboreum, G. hirsutum, and G. raimondii coding sequences were isolated as previously

121 described (Kerr et al. 2017). In brief, a BLAST query of the NCBI EST database was performed using

122 publicly available Arabidopsis AREB/ABF gene coding sequences for similar sequences from $G$. 
123 hirsutum to identify ESTs representing putative homologs. To recover full-length coding sequences,

124 total RNA from G. hirsutum (c.v Coker 312) was extracted using the Spectrum Plant Total RNA kit

125 (Sigma) and consecutive rounds of RACE-PCR were used to derive the 5' and 3' ends of the target

126 transcripts using the SMARTer RACE cDNA amplification kit (Clontech). G. arboreum and G.

127 raimondii $\mathrm{ABF}$ coding sequences were derived in a similar fashion for those sequences not found in the

128 NCBI database. Populus trichocarpa orthologs with the highest homology to Arabidopsis ABF1,

$129 A R E B 1 / A B F 2, A B F 3$, and AREB2/ABF4, as identified by Ji et al. (2013) and Brassica napus

$130 A R E B / A B F$ orthologs were obtained via BLAST queries of the NCBI database. Coding sequences were

131 imported into MEGA6.06-mac (Tamura et al., 2013), aligned with ClustalW, and used to generate a

132 maximum likelihood tree; bootstrapped 250 times. Aligned amino acid sequences were imported into

133 Jalview 2.9.Ob2 (Waterhouse et al., 2009) for annotation.

\section{5 qRT-PCR analysis}

136 Arabidopsis thaliana Columbia (Col-0) seeds, sown on solid medium containing half strength MS and

$1371 \%$ sucrose, were placed in the dark for $24 \mathrm{~h}$ at $4^{\circ} \mathrm{C}$, then transferred to a growth chamber at $24^{\circ} \mathrm{C}$ with

138 a $15 \mathrm{~h}$ light/9 h dark cycle for three weeks. Samples for basal expression level determination were

139 taken prior to stress treatments. To measure expression levels in response to exogenous ABA, plants

140 were sprayed to saturation with a $100 \mu \mathrm{M}$ ABA solution and sampled $0.5 \mathrm{~h}, 1 \mathrm{~h}$, and $2 \mathrm{~h}$ after

141 application. To measure the dehydration response, plants were removed from the media, keeping the

142 roots intact, and sampled after $1.5 \mathrm{~h}, 3 \mathrm{~h}$, and $6 \mathrm{~h}$. To measure the response to chilling temperatures,

143 plants were transferred to $4^{\circ} \mathrm{C}$, and sampled after $1 \mathrm{~h}, 2 \mathrm{~h}$, and $4 \mathrm{~h}$. G. hirsutum (Coker 312) plants were

144 grown in soil, in $1 \mathrm{~L}$ pots, for six to eight weeks under long-day conditions (15 h light/9 h dark) at

$14530^{\circ} \mathrm{C}$. Following pre-treatment sampling for the determination of basal expression, the plants were

146 subjected to the following treatments. Plants were sprayed to saturation with a $500 \mu \mathrm{M}$ ABA solution,

147 and sampled after $0.5 \mathrm{~h}, 1 \mathrm{~h}$, and $2 \mathrm{~h}$. Water was withheld, and dehydration stress treatment samples

148 were taken after $48 \mathrm{~h}$ (before visible wilting), $72 \mathrm{~h}$ (moderate wilting), and $78 \mathrm{~h}$ (severe wilting).

149 Chilling temperature treatment samples were taken after $1 \mathrm{~h}, 2 \mathrm{~h}$, and $4 \mathrm{~h}$ exposure to $4^{\circ} \mathrm{C}$. Arabidopsis

150 RNA was extracted using the RNeasy Mini kit (Qiagen). G. hirsutum RNA was extracted using the

151 Spectrum Plant Total RNA kit (Sigma). All RNA concentrations were quantified via Nanodrop,

152 normalized to $100 \mathrm{ng} / \mu \mathrm{L}$, and cDNA synthesis was performed using the iScript cDNA synthesis kit

153 (Bio-Rad). All qRT-PCR reactions were performed using the iTAQ Universal SYBR Green Supermix 
154 (Bio-Rad) in $10 \mu \mathrm{L}$ reactions. Standard curves were derived from pGWB12 plasmid constructs

155 (described in the following section) containing the Arabidopsis AREB/ABF or G. hirsutum ABF

156 homolog coding sequences.

\section{Generation of transgenic Arabidopsis lines}

159 The coding sequences of the $G$. hirsutum $A B F$ genes were amplified in accordance with the pENTR

160 Directional TOPO Cloning kit (Invitrogen). Half-reactions were used for TOPO cloning, then

161 transformed into One Shot Chemically Competent Escherichia coli (Invitrogen). Plasmids were

162 purified using the QIAprep Spin Miniprep kit (Qiagen). LR recombination (Invitrogen) was used to

163 transfer the target sequences to the pGWB12 expression vector (provided by T. Nakagawa, Research

164 Institute of Molecular Genetics, Shimane University, Matsue, Japan), then transformed into Library

165 Efficiency DH5- $\alpha$ E. coli (Invitrogen). Purified plasmid was transformed into Agrobacterium

166 tumefaciens $\mathrm{C} 58$, the culture was incubated at $30^{\circ} \mathrm{C}$ with shaking for $3 \mathrm{~h}$, then plated to solidified LB

167 supplemented with $10 \mu \mathrm{g} \mathrm{mL}^{-1}$ gentamicin, $50 \mu \mathrm{g} \mathrm{mL}^{-1}$ kanamycin, and $50 \mu \mathrm{g} \mathrm{mL}^{-1}$ rifampicin.

168 Colonies positive for the insert were cultured for $48 \mathrm{~h}$ in $25 \mathrm{~mL}$ liquid LB supplemented with $10 \mu \mathrm{g}$

$169 \mathrm{~mL}^{-1}$ gentamicin, $50 \mu \mathrm{g} \mathrm{mL}^{-1}$ kanamycin, and $50 \mu \mathrm{g} \mathrm{mL}^{-1}$ rifampicin at $30^{\circ} \mathrm{C}$ with shaking, then

170 transferred to $250 \mathrm{~mL} \mathrm{LB}$ for $24 \mathrm{~h}$. Cells were pelleted, then resuspended in a $400 \mathrm{~mL} 5 \%$ sucrose,

$1710.01 \%$ Silwet L-77 solution. Flowering Arabidopsis plants were dipped for $20 \mathrm{~s}$ with agitation, then

172 placed under cover in the dark for 24 hours before being transferred to growth conditions at $24{ }^{\circ} \mathrm{C}$ with

173 a 15 hour light/9 hour dark cycle (Clough and Bent, 1998). Harvested seeds were surface sterilized in

$17430 \%$ chlorine bleach and plated on solidified $1 / 2 \mathrm{MS}$ media containing $1 \%$ sucrose and $50 \mu \mathrm{g} \mathrm{mL}^{-1}$

175 kanamycin. Independent transformed lines were transferred to soil, verified via PCR, and expression

176 levels were measured using qRT-PCR (as above) for a minimum of ten lines. Three lines, the first

177 representing a relatively low level of ectopic expression, the second representing the highest level of

178 ectopic expression of the lines quantified, and the third, representing an approximate average

179 expression level of the low and high expressing lines (hereinafter "medial"), were selected for further

180 examination.

181

182 Immunoprecipitation

183 Immunoprecipitation assays were performed as previously described (Chen et al., 2013) with the

184 following modifications. Total protein from eight-day-old 35S::FLAG-GhABF expressing transgenic 
185 Arabidopsis seedlings was extracted in immunoprecipitation (IP) buffer (50 mM Tris- $\mathrm{HCl}$ (pH 8.1),

$186150 \mathrm{mM} \mathrm{NaCl}, 1 \% \mathrm{NP}-40$ (v/v), $1 \mathrm{mM}$ EDTA, 5\% glycerol, 1mM phenylmethylsufonyl fluoride, and

187 protease inhibitor cocktail (1:100)). The protein extracts (1 mg) were precleared by incubation with

188 Protein A/G beads (Santa Cruz) for $2 \mathrm{~h}$ at $4^{\circ} \mathrm{C}$, and immunoprecipitated using $20 \mu 1$ of Anti-FLAG

189 Affinity Gel (Sigma) at $4^{\circ} \mathrm{C}$ for $1 \mathrm{~h}$. Beads were washed three times with IP buffer for 20 min each at

$1904^{\circ} \mathrm{C}$. The precipitated proteins were eluted using 2x SDS sample buffer. Eluted samples were subjected

191 to Western blot analysis using an Anti-FLAG Alkaline Phosphatase antibody (Sigma). Each

192 experiment was replicated three times.

\section{Transgenic Arabidopsis development and abiotic stress tolerance evaluation}

195 To determine the effects of ectopic expression of the G. hirsutum ABF homologs in Arabidopsis on the 196 reproductive transition, three to four $\mathrm{T}_{3}$ generation transgenic plants were grown in soil in $15 \mathrm{ml}$ pots 197 alongside wild-type (WT) Arabidopsis. The reproductive transition, defined by the initiation of bolting, 198 was monitored for each transgenic line as compared to WT. To measure differential survival following 199 dehydration, homozygous $\mathrm{T}_{3}$ and WT seeds were surface sterilized in $30 \%$ bleach, plated on $1 / 2 \mathrm{MS}, 1 \%$ 200 sucrose solid medium, placed in the dark for 24 hours at $4{ }^{\circ} \mathrm{C}$, then transferred to a growth chamber at $20124^{\circ} \mathrm{C}$ with a $15 \mathrm{~h} \mathrm{light/9} \mathrm{h}$ dark cycle for 3 weeks. An average of ten plants from three plates for each 202 transgenic line and WT were removed from the media and transferred to petri dishes lined with glass 203 beads to dehydrate. Plants were re-watered, in $30 \mathrm{~min}$ intervals, after a minimum of $4 \mathrm{~h}$ dehydration, to 204 a maximum of $6.5 \mathrm{~h}$. Survival was recorded following a $48 \mathrm{~h}$ recovery period. Electrolyte leakage, as 205 the result of low water potential-induced damage, was measured as described by Verslues and Bray 206 (2004) and van der Weele et al. (2000), with minor modifications. Briefly, three-week-old seedlings 207 were transferred to PEG-infused plates of increasingly negative water potentials $(-0.25,-0.50,-0.75$, 208 and $-1.25 \mathrm{MPa}$ ) for $24 \mathrm{~h}$, rinsed in a mannitol solution of the same water potential, and placed in $5 \mathrm{~mL}$ 209 deionized water for $1 \mathrm{~h}$. Conductivity was measured, the samples were autoclaved, and conductivity 210 was measured again. Relative electrolyte leakage was calculated by dividing initial conductivity by 211 conductivity following autoclaving. Each genotype and treatment was replicated three times. To 212 measure differential survival following exposure to freezing temperatures, $\mathrm{T}_{3}$ seeds were sown on soil-

213 filled petri dishes. An average of ten 4 week-old plants per plate were then transferred to a growth

214 chamber at $-7^{\circ} \mathrm{C}$. After a minimum of $3 \mathrm{~h}$ at $-7^{\circ} \mathrm{C}$, plates were returned to the growth chamber at 215 approximately $24^{\circ} \mathrm{C}$, at $30 \mathrm{~min}$ intervals, to a maximum $5.5 \mathrm{~h}$. Survival was recorded following a $48 \mathrm{~h}$ 
216 recovery period. Electrolyte leakage as the result of freezing damage was measured as described by

217 Guo et al. (2002) and Ristic and Ashworth (1993), with minor modifications. Briefly, leaves of 4 week

218 old plants were excised and placed in tubes containing $5 \mathrm{~mL}$ deionized water then transferred to a water

219 bath at $1^{\circ} \mathrm{C}$. The temperature was decreased at a rate of $1.5^{\circ} \mathrm{C} \mathrm{h}^{-1}$ and samples were removed at $-2,-5$, -

2208 , and $-11^{\circ} \mathrm{C}$, and placed on ice overnight. Following the measurement of initial conductivity, the

221 samples were autoclaved, conductivity was measured again, and relative electrolyte leakage was

222 calculated. Each genotype and temperature was replicated three times.

\section{Results}

\section{Isolation and phylogenetic analysis of $G \boldsymbol{h A B F}$ homologs}

226 The allotetraploid $G$. hirsutum genome is a result of a polyploidy event between A and D genome

227 Gossypium diploid species (Wendel \& Cronn, 2003; Chaudhary et al., 2009). Therefore, we expected

228 that the target G. hirsutum ABF orthologs would occur in the G. hirsutum genome as highly similar,

229 albeit distinct, homeologous gene pairs. To confirm this hypothesis, we isolated the coding sequences

230 and portions of the promoter regions of multiple putative $A B F$ homologs from G. hirsutum and the

231 diploid Gossypium species, G. arboreum (A genome) and G. raimondii (D genome), and aligned them

232 with the Arabidopsis AREB/ABF orthologs (Supplementary Fig. S1). Eight putative polypeptides

233 encoding $G h A B F$ orthologs (four homeologous pairs) were derived that contained the conserved basic

234 region and leucine repeats requisite of the bZIP domain, and the five putative Ser/Thr phosphorylation

235 sites characteristic of the Arabidopsis AREB/ABFs (Furihata et al., 2006; Fujii et al., 2009).

237 To confirm the homology of these putative Gossypium orthologs, we constructed a maximum

238 likelihood phylogenetic tree (Supplementary Fig. S2) including the isolated G. arboreum, G. hirsutum,

239 and G. raimondii ABF coding sequences, their Arabidopsis and B. napus orthologs (Rosid II), and the $P$.

240 trichocarpa AREB/ABF homologs (Rosid I; Ji et al., 2013). Significant support was found for the

241 homology of the eight isolated $G h A B F$ sequences. Each of the Brassicaceae family $A R E B / A B F$

242 sequences resolved in a one-to-one fashion, as did the G. hirsutum homeologous pairs with their

243 corresponding A or D genome diploid Gossypium progenitor. However, no one-to-one $A R E B / A B F$ gene

244 relationship was found between the Malvaceae (Gossypium) and Brassicaceae families, or between the

245 Rosid I and Rosid II clades. The coding sequences of the Gossypium ABF homeologous pairs ABF1,

$246 A B F 3$, and $A B F 4$ were found to be more closely related to each other than to the $A R E B / A B F$ orthologs 
247 from any of the other genera examined, and also more closely related to two of the four $A R E B / A B F$

248 homologs from $P$. trichocarpa, rather than the examined species from the Rosid II clade, of which the

249 genus Gossypium is a member. Furthermore, the Gossypium ABF2 orthologs resolved with the

250 remaining two Rosid I clade homologs, and were more similar to the Arabidopsis $A B F 1, A B F 3$, and

251 AREB2/ABF4 and Gossypium ABF1, ABF3, and ABF4 homologs than to the corresponding

252 Arabidopsis AREB1/ABF2 homolog. Since no clear one-to-one phylogenetic orthologous relationship

253 was found between the Malvaceae (Gossypium) and Brassicacace species examined, we opted to label

254 the isolated $G h A B F$ homologs based on a combination of their phylogenetic relationships and

255 similarities to the Arabidopsis AREB/ABFs in their expression patterns in response to abiotic stress (as

256 described in the following section).

\section{The $A t A R E B / A B F s$ and $G h A B F s$ are differentially expressed in response to abiotic stress}

259 The $A R E B / A B F s$ have been widely reported to be differentially expressed in response to various abiotic 260 stressors in several plant species (Choi et al., 2000; Fujita et al., 2005; Orellana et al., 2012; Li et al., 261 2014; Yoshida et al., 2015). Therefore, we used qRT-PCR to measure the expression patterns of the 262 Arabidopsis $A R E B / A B F$ and $G h A B F$ homologs in response to exogenous ABA application, water 263 deficit, and cold temperature stress (Figs. 1 and 2). Analyses of the Arabidopsis homologs was carried 264 out to provide baseline expression level data to which the GhABF expression levels could be compared. 265 Absolute quantification methods were used to measure transcript copy number so that expression 266 changes between the different genes could be compared directly. Relative quantification was also 267 performed to confirm that our results were consistent with previously published data (Choi et al., 2000; 268 Kim et al., 2004; Fujita et al., 2005; Oh et al., 2005; Yoshida et al., 2015). We found basal expression 269 levels of the Arabidopsis AREB/ABFs ranged from an average low of 10 transcripts per ng total RNA 270 for $A t A B F 1$, to 21 and 27 copies for $A t A R E B 1 / A B F 2$, and $A t A B F 3$ and AtAREB2/ABF4, respectively

271 (Fig. 1A,C,E). Similar low levels of basal expression were measured for the $G h A B F$ homologs, ranging 272 from an average of 2 copies per ng total RNA for $G h A B F 1 D$, to an average of 18 copies for $G h A B F 2 A$ 273 and GhABF4D (Fig. 2).

275 As previously reported, we found the Arabidopsis $A R E B / A B F$ homologs were differentially expressed 276 in response to exogenous $\mathrm{ABA}$ and abiotic stress treatments. While expression of each AtAREB/ABF 277 gene was induced, at least to some degree, in response to exogenous ABA application, the magnitude of 
increase differed substantially. AtABF1 expression doubled and $A t A R E B 1 / A B F 2$ expression tripled

relative to basal levels, while the expression of $A t A B F 3$ increased 6 fold and AtAREB2/ABF4 increased

7 fold (Fig. 1A,B). Similarly, all Arabidopsis AREB/ABF genes were induced in response to water deficit, though $A t A B F 1$ and $A t A R E B 1 / A B F 2$ transcript levels increased only slightly, while the AtAREB2/ABF4 transcript level increased steadily to 20 times its basal level over the $6 \mathrm{~h}$ sampling period, and the $A t A B F 3$ level increased quickly after $3 \mathrm{~h}$ to ultimately reach a level 75 fold greater than the basal level after $6 \mathrm{~h}$ (Fig. 1C,D). Though the $A R E B / A B F s$ genes are primarily associated with the response to drought via the ABA-dependent pathway (Lee et al., 2010; Fujita et al., 2013; Yoshida et $a l ., 2014)$, we also examined their expression in response to low temperature stress. The expression of AtABF 1 and AtAREB2/ABF4 did not change in response to chilling, however, the AtAREB1/ABF2 transcript level increased gradually to 3 times its basal expression over $4 \mathrm{~h}$ at $4{ }^{\circ} \mathrm{C}$, and $A t A B F 3$ expression rose quickly to 6 six times its basal level after $1 \mathrm{~h}$ at $4{ }^{\circ} \mathrm{C}$, then declined after the $2 \mathrm{~h}$ time

The expression of each $G h A B F$ homeolog was induced in response to at least one stress treatment, though the magnitude of induction varied widely between treatments (Fig. 2), and no consistent bias in expression of the A or D genome was observed. While expression of each of the eight $G h A B F$ homologs increased in response to exogenous $\mathrm{ABA}$ application, induction of $G h A B F 3 A$ was by far the strongest, rising to a level 30 times its basal expression over the course of the $2 \mathrm{~h}$ assay. Expression of $G h A B F 3 D$ and both $G h A B F 4$ homeologs increased more gradually in response to ABA, reaching levels 8 to 10 fold above basal levels, while expression of the GhABF2 homeologs increased by about 5 fold, and the GhABF1 homeologs increased only by about 2 to 3 fold during the $2 \mathrm{~h}$ assay (Fig. 2A-D). In response to water deficit stress, again, $G h A B F 3 A$ expression showed the largest increase in transcript copy number. In addition, the increase in expression of the GhABF3 homeologs in response to water deficit treatment began earlier than the other $G h A B F$ genes, becoming apparent after $48 \mathrm{~h}$, as compared to $72 \mathrm{~h}$ for the $G h A B F 1, G h A B F 2$, and GhABF4 homeologous gene pairs (Fig. 2E-H). Furthermore,

304 both ABA- and drought-induced expression of $G h A B F 3 A$ was considerably stronger than that of $305 G h A B F 3 D$, illustrating differential expression among these homeologous pairs.

307 Again, while the AREB/ABF bZIP transcription factors are not generally associated with temperature 308 stress, we found the expression of $A t A B F 2$ and $A t A B F 3$, and at least one member of each $G h A B F$ 
309 homeologous gene pair, was induced during exposure to low temperature, although the magnitude of

310 change exhibited by most of these $G h A B F$ homologs was far less than in the exogenous ABA

311 application or water deficit treatments (Fig. 2I-L). Transcript levels of most of the GhABF genes

312 induced by low temperature reached a maximum after $1 \mathrm{~h}$ at $4{ }^{\circ} \mathrm{C}$, then leveled off or dropped back to

313 near basal levels over the duration of the treatment. GhABF1A, which showed a relatively weak

314 response to $\mathrm{ABA}$ or water deficit stress, was the most strongly induced $G h A B F$ homolog in response to

315 low temperature, increasing from single digit levels to more than 100 copies per ng total RNA within 1

$316 \mathrm{~h}$, before returning to near basal levels after $4 \mathrm{~h}$. Expression of $G h A B F 4 D$ also increased considerably

317 in response to chilling stress, and like GhABF1A, expression returned to near basal levels after $4 \mathrm{~h}$.

319 Generation of $\mathrm{GhABF}$ expressing transgenic Arabidopsis lines

320 In order to characterize the functions of the individual GhABF homeologs and test the impact of their

321 ectopic expression on development and abiotic stress tolerance, we generated independent transgenic

322 Arabidopsis lines that ectopically express each of the eight isolated GhABF genes, under the control of

323 the constitutive CaMV 35S promoter. The ectopic expression levels of a minimum of ten independent

324 Arabidopsis lines for each gene construct were quantified, and three lines for each were selected for

325 phenotypic examination. These transgenic lines were selected as per the following crteria: 1) the line

326 with the lowest measurable ectopic expression level, 2) the line with the highest measured ectopic

327 expression level, and 3) a line with an ectopic expression level approximating the midpoint between the

328 high and low expressing lines for each gene construct (Table1). Each of these three selected lines, from

329 each of the eight $G h A B F$ gene constructs, were subsequently evaluated, in parallel, for differences in

330 growth and development, and their ability to tolerate drought and low temperature stress.

332 Although the same binary vector and CaMV35S promoter were used in the generation of all gene

333 constructs, we found substantial differences in the levels of constitutive ectopic expression among the

334 independent transgenic Arabidopsis lines. Wide variation in the range of event-specific expression was

335 seen between the transgenic lines expressing the individual GhABF orthologs and, in some cases,

336 between the lines expressing the A or D genome-derived homeologs (Table 1). For example, the high-

337 expressing lines containing the transgenes that encode the GhABF2 A and D genome homeologs had

338 similar levels of expression, averaging 455 transcripts per ng total RNA, while the GhABF2A-

339 expressing lines showed little event-specific variability, with less than a 2-fold difference detected 
340 between the highest and lowest expressing lines, in contrast to the difference between highest and 341 lowest expressing $G h A B F 2 D$ lines, which was nearly 16-fold. Greater event-specific variation in 342 expression was seen in the GhABF3 homeolog expressing lines, with the selected $G h A B F 3 A$ lines 343 ranging from a low of 175 transcripts per ng total RNA to a high of 6383 transcripts per ng total RNA,

344 a 36-fold difference, while the overall expression difference among the GhABF3D lines was 345 approximately $1 / 10^{\text {th }}$ the level of the GhABF3A lines, ranging from 17 to 770 transcripts per ng total 346 RNA, a 45-fold difference from lowest to highest. Even more substantial differences in expression 347 between the paired homeologs was seen among the GhABF4 lines, with the expression of the 348 GhABF4A lines ranging from 224 to 1563 transcripts per ng RNA (a 7-fold difference), while 349 expression levels in the GhABF4D lines were far lower, ranging from 22 to 63 transcripts per ng RNA, 350 a difference of only about 3 -fold. Thus, in addition to the expected event-specific variation in transgene expression that is typically attributed to position effects associated with the insertion site, substantial gene-specific differences in mRNA accumulation are also apparent.

\section{Gh ABF protein expression is largely independent of transcript level}

355 To better understand the patterns of ectopic G. hirsutum ABF expression in Arabidopsis and determine the effects of ABA on ABF accumulation (Chen et al., 2013) we examined FLAG-GhABF fusion protein accumulation in the selected $G h A B F \mathrm{D}$ genome expressing transgenic Arabidopsis lines with or without ABA treatment (Fig. 3). Ectopic GhABF protein expression was not detected in crude protein extracts from any of our transgenic lines by Western blot analysis but specific bands were detectable after enrichment by immunoprecipitation. Unlike the wide variation in transcript expression levels, relatively little variation in G. hirsutum $\mathrm{ABF}$ protein accumulation was seen between the low, median, and high transcript expressing $G h A B F 2 D$ or $G h A B F 4 D$ transgenic Arabidopsis lines without ABA

363 treatment and these levels did not change in response to ABA. In contrast, GhABF3D protein levels were nearly undetectable in immunoprecipitated samples taken from plants without ABA treatment but, after $\mathrm{AB} A$ treatment, the protein accumulated to substantially higher levels and clear differences were seen between the low, median, and high expressing lines. Thus, it appears that the steady state levels of

367 the $G h \mathrm{ABF}$ proteins in plants that express $G h A B F 2 D$ and $G h A B F 4 D$ are relatively stable and largely 368 independent of transcript levels or $\mathrm{ABA}$ treatment. On the other hand, accumulation of GhABF3D appears to be under ABA-dependent post-transcriptional regulation. 


\section{Ectopic $G h A B F$ expression can delay the reproductive transition}

372 Previous studies have shown that endogenous ectopic expression of Arabidopsis AREB/ABFs delays

373 growth and the reproductive transition (Kang et al., 2002; Kim et al., 2004; Fujita et al., 2005). To

374 determine if ectopic GhABF gene expression in Arabidopsis affects development, selected transgenic

375 lines were grown alongside wild type and monitored for differences in the reproductive transition,

376 defined by the initiation of bolting (Fig. 4). None of the GhABF1A or GhABF1D expressing lines

377 examined differed significantly from wild type plants; however, the majority of the $G h A B F 2, G h A B F 3$,

378 and GhABF4 transgenic Arabidopsis lines exhibited significant delays in reproductive transition (Fig.

379 4B). Except for the $G h A B F 1$ expressing lines, the reproductive transition delay was most severe the

380 lines that express the highest ectopic levels of the $G h A B F$ transcripts, indicating a relationship between

381 expression level and reproductive delay. For example, while GhABF2D lines showed some line to line

382 variation in mRNA expression, the level of GhABF2D protein was relatively stable and this is reflected

383 in a limited range of developmental delay phenotypes. Likewise, expression of GhABF4D mRNA was

384 low but protein accumulation in these lines was relatively high and stable, which corresponds with the

385 strong developmental delay in all three lines. On the other hand, GhABF3D lines showed strong

386 variation in expression at the mRNA level and, following ABA treatment, at the protein level. Thus,

387 not unexpectedly, the severity of developmental delay in $G h A B F$ expressing Arabidopsis plants appears

388 to correlate more closely with $G h A B F$ transgene expression at the protein level than at the mRNA level.

390 Ectopic $\boldsymbol{G h A B F}$ expression can improve tolerance to water deficit and osmotic stress

391 To determine if ectopic GhABF expression in Arabidopsis confers improved water deficit tolerance, we 392 quantified the survival of the selected GhABF expressing transgenic Arabidopsis lines, as compared to 393 wild type, following dehydration treatment (Fig. 5A). Substantial differences in survival were apparent

394 between the wild type and transgenic plants after approximately $5.5 \mathrm{~h}$ dehydration, and these

395 differences became more pronounced after 6 h (Fig. 5A; Supplementary Table S1). The percent of

396 surviving plants corresponded with ectopic expression level in the majority of the $G h A B F$-expressing

397 lines, with the strongest protective effects seen in the high expressing lines for most gene constructs.

398 Notable exceptions to this trend were seen in the GhABF4 expressing plants, which showed similar

399 survival rates at all expression levels. While survival of the GhABF4A plants was not substantially

400 higher than wild type despite relatively high levels of ectopic expression, GhABF4D lines showed

401 significantly improved survival that correlated more closely with the expression at the protein level. 
402 The most substantial increase in water deficit tolerance was seen in the high GhABF3D line, which

403 showed $71 \%$ survival over wild type after $6 \mathrm{~h}$ dehydration treatment, and correlated most closely with

404 protein expression levels after ABA treatment.

406 To corroborate the dehydration survival assay results with osmotic stress, each of the $G h A B F$ -

407 expressing lines were subjected to increasingly negative water potentials, and the percent electrolyte

408 leakage was measured (Fig. 5B, Supplementary Table S2). Ectopic expression of the GhABF homologs

409 resulted in reduced electrolyte leakage in nearly all of the lines and, in the majority of the transgenic

410 lines, reduced electrolyte leakage following osmotic stress corresponded with increased plant survival

411 following dehydration. With the exception of the GhABF1A and GhABF2D lines, the highest

412 expressing lines showed the lowest levels of electrolyte leakage. However, this trend was not

413 proportional to the dehydration survival results in all cases. For example, all of the $G h A B F 3 A$

414 expressing lines showed substantially reduced membrane damage, which contrasts with the plant

415 survival assay, in which the low and medial expressing lines performed similarly to wild type.

416 Likewise, the GhABF4A and GhABF4D transgenic lines examined exhibited similar survival rates (by

417 homeolog) regardless of expression level, but lines with increasing levels of ectopic expression showed

418 incremental reductions in electrolyte leakage. The GhABF3D lines, on the other hand, showed both

419 substantial increases in survival and substantial reductions in electrolyte leakage corresponding most

420 closely to the level of ectopic expression at the protein level.

422 Overall, these results indicate that ectopic expression of each of the GhABF homologs in Arabidopsis

423 resulted in protective effects in at least one of the assays used and the magnitude of stress protection

424 was related to transgene expression level in the $G h A B F 1, G h A B F 2$, and $G h A B F 3$ expressing lines.

425 However, in the GhABF4 expressing lines, little correlation was evident between transgene expression

426 level and stress protection in the dehydration survival assay, where the highest expressing $G h A B F 4 A$

427 line, which had transcript levels approximately 25-times higher than highest expressing the GhABF4D

428 line, was much more sensitive to dehydration stress. However, as shown in Fig 3, GhABF4D plants

429 accumulate relatively high levels of GhABF4D protein, in spite of showing relatively low levels of 430 mRNA.

431

432 Ectopic $\boldsymbol{G h A B F}$ expression can improve cold tolerance, in a gene dependent manner 
433 Although the $A R E B / A B F s$ are generally associated with the osmotic stress response, some studies

434 indicated they can also influence cold responses, directly or indirectly, via crosstalk with cold-

435 responsive signaling pathways (Choi et al., 2000; Oh et al., 2005; Lee et al., 2010; Fujita et al., 2011).

436 Therefore, to determine if ectopic GhABF gene expression in Arabidopsis has an effect on cold

437 tolerance, we analyzed survival following exposure to $-7^{\circ} \mathrm{C}$ over the course of $5 \mathrm{~h}$ (Fig. 6A;

438 Supplementary Table S3), and electrolyte leakage (Fig. 6B, Supplementary Table S4) in response to

439 progressively lower freezing temperatures.

441 Unlike the water deficit tolerance assays where the protective effects were associated with expression

442 level, the effects of ectopic expression of the G.hABF homologs in Arabidopsis on freezing temperature

443 survival were gene-specific and largely independent of expression at the mRNA level (Fig. 6A). For

444 example, all of the transgenic Arabidopsis lines expressing either the GhABF1 or GhABF4 homeologs

445 showed significant increases in survival following exposure to $-7^{\circ} \mathrm{C}$ as compared to the wild type

446 plants. However, the ectopic expression of the $G h A B F 2 A$ and $G h A B F 3 A$ appeared to have negative

447 effects on freezing tolerance, and only plants that expressed high levels of $G h A B F 2 D$ or $G h A B F 3 D$

448 showed increased survival compared to wild type plants.

450 Similar to the water deficit stress assays, lower levels of electrolyte leakage following exposure to 451 freezing temperatures generally correlated with increased plant survival (Fig. 6B). Relative to wild type 452 plants, the percent of electrolyte leakage measured for all GhABF1 and $G h A B F 4$ lines examined was 453 substantially reduced, indicating enhanced cellular tolerance to freezing temperatures. Conversely, 454 expression of $G h A B F 2 A, G h A B F 2 A$, and $G h A B F 3 A$ appeared to result in significant increases in 455 electrolyte leakage after freezing treatment, relative to the wild type plants. Although plants of the high 456 expressing $G h A B F 2 D$ line showed a small but significant increase in survival, electrolyte leakage assay 457 results show that these plants suffered membrane damage similar to the wild type plants. High 458 expressing $G h A B F 3 A$ line and the low and medial expressing $G h A B F 2 A$ lines showed both reduced 459 survival and increased electrolyte leakage after exposure to freezing temperatures, indicating that 460 freezing tolerance in these plants is likely to be reduced. In summary, ectopic expression of either of 461 the GhABF1A, GhABF1D, and GhABF4D homeologs in Arabidopsis conferred increased tolerance to 462 freezing temperatures while expression of $G h A B F 2 A$ or $G h A B F 3$ homeologs appears to compromise 463 freezing tolerance. 


\section{Discussion}

466 To determine the functional roles of the $G h A B F$ orthologs, we examined their expression patterns in 467 response to various abiotic stressors in cotton and evaluated their effects on development and abiotic 468 stress tolerance by ectopically expressing each in Arabidopsis. Since G. hirsutum is an allotetraploid 469 species, we anticipated that each $G h A B F$ ortholog would be present in the cotton genome as a 470 homeologous pair of genes with very similar coding sequences. Eight $G h A B F$ coding sequences were 471 isolated, each encoding a putative polypeptide that contains the defining features of the Arabidopsis 472 AREB/ABF proteins, namely, a canonical bZIP domain, and five Ser/Thr kinase phosphorylation sites 473 (Furihata et al., 2006; Fujii et al., 2009). In order to directly compare the expression characteristics of 474 the individual $G h A B F$ genes to one another and to the AREB/ABF homologs from Arabidopsis,

475 absolute quantification methods were used to determine the number of transcript copies present in total 476 RNA samples. Furthermore, since the responses of the Arabidopsis AREB/ABFs to cold stress have 477 only been analyzed in a few cases (Choi et al., 2000; Lee et al., 2005), we assayed the expression of 478 these gene in response to low temperatures, in addition to exogenous ABA application and water 479 deficit. We found both the Arabidopsis $A R E B / A B F$ and $G h A B F$ genes had low levels of basal expression, and each gene was differentially responsive to the various abiotic stress treatments.

482 In Arabidopsis, expression of $A t A B F 3$ is the most responsive to water deficit, chilling temperatures and, 483 along with AtABF4, to ABA treatment, while in G. hirsutum, expression of the GhABF3A is the most 484 highly responsive homeolog to water deficit and ABA treatment, and GhABF $1 A$ is most responsive to 485 chilling. These differential expression patterns within the G. hirsutum homeologous pairs could 486 indicate sub-functionalization or silencing of one or the other homeolog due to redundancy. For 487 example, expression of $G h A B F 1$ homeologs was only modestly responsive to exogenous $\mathrm{ABA}$ or 488 dehydration, and the GhABF4 genes exhibit only a slight induction in response to dehydration, 489 however, GhABF1A and GhABF4D are strongly induced in response to chilling, while expression of $490 G h A B F 1 D$ responds relatively weakly to chilling and GhABF4A does not respond at all. This increased 491 expression in response to chilling stress could result from cross-talk due to functional interactions 492 between the ABA-dependent and ABA-independent stress response pathways (Yoshida et al., 2014). 493 For example, Arabidopsis AREB1/ABF2 interacts with various AP2 domain proteins, including 494 DREB1A, also known as CBF3, an essential component of the low temperature stress response (Lee et 
al., 2010, Zhou et al., 2011).

497 While ectopic expression of $A R E B / A B F$ genes may confer increased stress tolerance, these

498 improvements are often accompanied by delayed growth or reproduction (Kang et al., 2002; Kim et al.,

499 2004; Fujita et al., 2005). Therefore, we analyzed the ability of the GhABFs to confer increased stress

500 tolerance and affect development when ectopically expressed in Arabidopsis. Tradeoffs between stress

501 tolerance and developmental delay were seen with some, but not all, GhABF genes, raising the

502 possibility that negative side-effects on growth and development associated with increased $A R E B / A B F$

503 expression may be gene-specific and it might be possible to mitigate unwanted negative effects by

504 using transgenes that encode specific $A B F$ orthologs and selecting transgenic lines with varying levels

505 of ectopic expression. In this way, it may be possible to find an acceptable balance between positive

506 and negative phenotypes. Therefore, three independent lines with high, low, and medial levels of

507 ectopic expression were selected for each of the eight $G h A B F$ gene constructs for physiological

508 examination. Although the gene constructs differed only in their coding sequences, transgene

509 expression levels varied widely among the different $G h A B F$ gene constructs. For example, the highest

510 expressing GhABF3A line accumulated more than 6300 transcript copies/ng of total RNA and the

511 medial expressing line had higher transcript levels than the highest expressing line of any of the other

512 constructs. On the other hand, the highest expressing GhABF4D line produced only 63 copies/ng,

$5131 / 100^{\text {th }}$ of the level seen in the high expressing GhABF3A line. Yet, these transgenic lines showed

514 similar dehydration stress tolerance phenotypes and the $G h A B F 4 D$ line flowered later and showed

515 stronger cold tolerance than the high expressing GhABF3A line.

517 The large transgene-specific and event-specific differences in the steady-state levels of the ectopic

$518 G h A B F$ transcripts in plants of various transgenic lines does not seem to correspond well with the stress

519 tolerance phenotypes of these lines. A possible explanation for this paradox becomes apparent when

520 protein expression levels are considered. Regardless of the level of mRNA expression, only a very

521 small amount of GhABF protein accumulates in any of the transgenic Arabidopsis plants, as indicated

522 by the requirement for immunoprecipitation to allow detection. This suggests that accumulation of

$523 G h \mathrm{ABF}$ gene products is under strong post-transcriptional regulation. Chen et al. (2013) reported that

524 AtABF1 and AtABF3 turnover rapidly in the absence of ABA, and degradation is slowed when the

525 plants are pre-treated with $\mathrm{ABA}$ and our results indicate that accumulation of GhABF3D is $\mathrm{ABA}$ 
dependent. Thus, ABA appears to play a role in both the transcriptional and post-transcriptional

527 regulation of some AREB/ABFs in both Arabidopsis and G. hirsutum, while protein accumulation in

$528 G h A B F 2 D$ and $G h A B F 3 D$ lines appears to be relatively insensitive to the levels of mRNA and does not

529 respond to ABA treatment.

531 The effect of ectopic GhABF gene expression on cold tolerance in Arabidopsis follows a different

532 pattern to that observed for developmental delay and dehydration tolerance. There are few apparent

533 intragenic or intergenic expression level effects, in fact, the cold tolerance phenotype of the low

534 expressing $G h A B F 4 D$ lines is stronger than the much more highly expressed $G h A B F 4 A$ lines. However,

535 as with the other characteristics, expression of genes within the homeologous gene pairs generally

536 show similar phenotypes. Interestingly, all GhABF1A and GhABF1D expressing lines showed

537 substantially increased cold tolerance but no reproductive delay, while the improved cold tolerance of

$538 G h A B F 4 A$ and $G h A B F 4 D$ expressing lines was associated with severe reproductive delays.

540 Though possible, it seems unlikely that the large gene-specific differences in transcript abundance

541 result from position effects associated with the stochastic insertion of transgenes into the Arabidopsis

542 genome. It seems more probable that the differences in maximal transgene expression are due to the

543 characteristics of the individual G. hirsutum ABF coding sequences. These differences could affect

544 transcription, but it is more likely that they affect transcript stability. For example, the attenuating

545 effects of microRNA (miRNA) could differentially affect the accumulation of GhABF mRNA from

546 different transgenes. To examine this possibility, the coding sequences of the eight G. hirsutum ABF

547 homologs were used to query the Arabidopsis miRNA collection in miRBase. Between two and five

548 potential miRNA target sites were found within the coding sequences for the all of the G. hirsutum

$549 A B F s$, with the exception of the $G h A B F 3$ homeologs, for which no putative target sites were found.

550 This observation raises the possibility that the high levels of ectopic expression of the $G h A B F 3$

551 homeologs in transgenic Arabidopsis lines could be associated with differential sensitivity to miRNA-

552 dependent transcript destabilization. On the other hand, a unique potential miRNA target site was

553 detected in the GhABF4D coding sequence, which might explain its low expression. Interestingly, this

554 miRNA was reported to target transcripts for a MYB transcription factor that interacts with a class of

555 ABRE elements in the promoter of the stress responsive RD22 gene of Arabidopsis (Choi et al., 2000).

556 The possible direct or indirect effects of this or other miRNAs on $G h A B F$ transcript stability remain to 
557 be investigated.

559 Overall, our results indicate the isolated $G h A B F$ homologs encode functional transcription factors that

560 are likely to play important roles in the regulation of abiotic stress tolerance in cotton. Each homeolog

561 is differentially expressed in response to various abiotic stressors, and the ectopic expression of the

562 majority of these genes confers some degree of increased tolerance to drought or cold stress in

563 Arabidopsis. Keeping in mind that these results represent phenotypic analyses of transgenic

564 Arabidopsis plants that ectopically express cotton $A B F$ genes, it is clear that $G h A B F 3$ genes are

565 induced by ABA and dehydration at both the transcriptional and post-transcriptional levels, and

566 together with the GhABF4 genes, may be critical for controlling cellular responses to water deficit in

567 cotton. Likewise, since ectopic expression of the GhABF1 and GhABF4 homeologs provides

568 substantial increases in cold tolerance in Arabidopsis, it seems possible that these factors may also be

569 important for the regulation of cold responsive gene expression in cotton. These data provide a

570 tentative roadmap toward informed decisions regarding the selection of genes for the development of

571 transgenic plants aimed at improving abiotic stress tolerance. However, further functional analyses of

572 the expression of these transgenes in other species, including cotton, will be necessary to confirm these

573 preliminary conclusions. 


\section{Supplementary data}

576 Table S1. Percent survival of selected GhABF expressing transgenic Arabidopsis lines after 5.5 and $6 \mathrm{~h}$ 577 dehydration.

578 Table S2. Electrolyte leakage (\%) of selected GhABF expressing transgenic Arabidopsis lines in

579 response to increasingly negative water potentials

580 Table S3. Percent survival of selected GhABF expressing transgenic Arabidopsis lines after 4.5 and 5

581 hours at $-7^{\circ} \mathrm{C}$.

582 Table S4. Electrolyte leakage (\%) of selected GhABF expressing transgenic Arabidopsis lines in

583 response to increasingly negative temperatures.

584 Fig. S1. Multiple sequence alignment of the Arabidopsis AREB/ABFs and GhABFs.

585 Fig. S2. Maximum likelihood tree of select AREB/ABF subfamily members.

586

\section{Acknowledgments}

588 The authors thank Drs. Mohamed Fokar, Miyoung Kang, and Paxton Payton for experimental

589 consultation and critical reviews of the manuscript. We also thank Drs. Andrew Doust, Million Tadege,

590 and Ramanjulu Sunkar for helpful discussions. This work was supported by grants from Cotton

591 Incorporated and the Samuel Roberts Noble Foundation to RDA, along with additional support from

592 the Walter R. Sitlington Endowment, the Oklahoma Agricultural Experiment Station, and the

593 Department of Biochemistry and Molecular Biology, Oklahoma State University. 
594 References

595 Bartels D, Sunkar R. 2005. Drought and salt tolerance in plants. Critical Reviews in Plant Sciences

596 24, 23-58.

597 Boyer JS. 1982. Plant productivity and environment. Science 218, 443-448.

598 Bray EA, Bailey-Serres J, Weretilnyk E. 2000. Responses to abiotic stresses. In: Buchanan BB,

599 Gruissem W, Jones, RL, eds. Biochemistry and Molecular Biology of Plants. Rockville, Maryland:

600 American Society of Plant Physiologists, 1158-1249.

601 Chaudry B, Hovav R, Flagel L, Mittler R, Wendel JF. 2009. Parallel expression evolution of

602 oxidative stress-related genes in fiber from wild and domesticated diploid and polyploid cotton

603 (Gossypium). BMC Genomics 10, 378-390.

604 Chinnusamy V, Zhu J, Zhu JK. 2006. Gene regulation during cold acclimation in plants. Physiologia 605 Plantarum 126, 52-61.

606 Choi HI, Hong JH, Ha JO, Kang JY, Kim SY. 2000. ABFs, a family of AB A-responsive element

607 binding factors. Journal of Biological Chemistry 275, 1723-1730.

608 Clough SJ, Bent AF. 1998. Floral dip: a simplified method for Agrobacterium- mediated

609 transformation of Arabidopsis thaliana. The Plant Journal 16, 735-743.

610 Fujii H, Chinnusamy V, Rodrigues A, Rubio S, Antoni R, Park S, Cutler SR, Sheen J, Rodriguez

611 PL, Zhu, J. 2009. In vitro reconstitution of an abscisic acid signalling pathway. Nature 462, 660-664.

612 Fujita Y, Fujita M, Satoh R, Maruyama K, Parvez MM, Seki M, Hiratsu K, Ohme-Takagi M,

613 Shinozaki K, Yamaguchi-Shinozaki, K. 2005. AREB1 is a transcription activator of novel ABRE-

614 dependent ABA signaling that enhances drought stress tolerance in Arabidopsis. The Plant Cell 17, $615 \quad 3470-3488$.

616 Fujita Y, Yoshida T, Yamaguchi-Shinozaki K. 2013. Pivotal role of the AREB/ABF-SnRK2 pathway 617 in ABRE-mediated transcription in response to osmotic stress in plants. Physiologia Plantarum 147, 1561827.

619 Furihata T, Maruyama K, Fujita Y, Umezawa T, Yoshida R, Shinozaki K, Yamaguchi-Shinozaki

620 K. 2006. Abscisic acid-dependent multisite phosphorylation regulates the activity of a transcription 621 activator AREB1. Proceedings of the National Academy of Sciences USA 103, 1988-1993.

622 Guo Y, Xiong L, Ishitani M, Zhu J. 2002. An Arabidopsis mutation in translation elongation factor 2 623 causes superinduction of CBF/DREB1 transcription factor genes but blocks the induction of their 624 downstream targets under low temperature. Proceedings of the National Academy of Sciences USA 99, 
7786-7791.

626 Ji L, Wang J, Ye M, Li Y, Guo B, Chen Z, Li H, An X. 2013. Identification and characterization of 627 the Populus AREB/ABF subfamily. Journal of Integrative Plant Biology 55, 177-186.

628 Kang JY, Choi HI, Im MY, Kim SY. 2002. Arabidopsis basic leucine zipper proteins that mediate 629 stress-responsive abscisic acid signaling. The Plant Cell 14, 343-357.

630 Kerr TCC, Abdel-Mageed H, Alemant L, Lee J, Payton P, Cryer D, Allen AD. 2017. Ectopic 631 expression of two AREB/ABF orthologs increases drought tolerance in cotton (Gossypium hirsutum).

632 Plant, Cell and Envirnment doi: 10.1111/pce.12906

633 Kim S, Kang JY, Cho DI, Park JH, Kim SY. 2004. ABF2, an ABRE-binding bZIP factor, is an 634 essential component of glucose signaling and its overexpression affects multiple stress tolerance. The 635 Plant Journal 40, 75-87.

636 Lee B, Henderson DA, Zhu J. 2005. The Arabidopsis cold-responsive transcriptome and its regulation 637 by ICE1. The Plant Cell 17, 3155-3175.

638 Lee SJ, Kang JY, Park HJ, Kim MD, Bae MS, Choi HI, Kim SY. 2010. DREB2C interacts with $639 \mathrm{ABF}$, a bZIP protein regulating abscisic acid-responsive gene expression, and its overexpression 640 affects abscisic acid sensitivity. Plant Physiology 153, 716-727.

641 Li C, Yue J, Wu X, Xu C, Yu J. 2014. An ABA-responsive DRE-binding protein gene from Setaria 642 italica, SiARDP, the target gene of SiAREB, plays a critical role under drought stress. Journal of 643 Experimental Botany 65, 5415-5427.

644 Medina J, Catalá R, Salinas J. 2011. The CBFs: three Arabidopsis transcription factors to cold 645 acclimate. Plant Science 180, 3-11.

646 Novillo F, Medina J, Salinas J. 2007. Arabidopsis CBF1 and CBF3 have a different function than $647 \mathrm{CBF} 2$ in cold acclimation and define different gene classes in the CBF regulon. Proceedings of the 648 National Academy of Sciences USA 104, 21002-21007.

649 Oh S, Song SI, Kim YS, Jang H, Kim SY, Kim M, Kim Y, Nahm BH, Kim J. 2005 Arabidopsis $650 \mathrm{CBF} / \mathrm{DREB} 1 \mathrm{~A}$ and $\mathrm{ABF} 3$ in transgenic rice increased tolerance to abiotic stress without stunting 651 growth. Plant Physiology 138, 341-351.

652 Orellana S, Yanez M, Espinoza A, Verdugo I, Gonzalez E, Ruiz-Lara S, Casaretto JA. 2010. The 653 transcription factor SIAREB1 confers drought, salt stress tolerance and regulates biotic and abiotic 654 stress-related genes in tomato. Plant, Cell \& Environment 33, 2191-2208.

655 Osakwe E. 2009. Cotton Fact Sheet India. International Cotton Advisory Committee. 
Reddy AR, Chaitanya KV, Vivekanandan M. 2004. Drought-induced responses of photosynthesis and antioxidant metabolism in higher plants. Journal of Plant Physiology 161, 1189-1202.

658 Ristic Z, Ashworth EN. 1993. Changes in leaf ultrastructure and carbohydrates in Arabidopsis

659 thaliana (Heynh) cv. Columbia during rapid cold acclimation. Protoplasma 172, 111-123.

660 Tamura K, Stecher G, Peterson D, Filipski A, Kumar S. 2013. MEGA6: Molecular Evolutionary

661 Genetics Analysis Version 6.0. Molecular Biology and Evolution 30, 2725-2729.

662 van der Weele CM, Spollen WG, Sharp RE, Baskin TI. 2000. Growth of Arabidopsis thaliana

663 seedlings under water deficit studied by control of water potential in nutrient-agar media. Journal of

664 Experimental Biology 51, 1555-1562.

665 Verslues PE, Bray EA. 2004. LWR1 and LWR2 are required for osmoregulation and osmotic

666 adjustment in Arabidopsis. Plant Physiology 136, 2831-2842.

667 Wang W, Vinocur B, Altman A. 2003. Plant responses to drought, salinity and extreme temperatures:

668 towards genetic engineering for stress tolerance. Planta 218, 1-14.

669 Waterhouse AM, Procter JB, Martin DMA, Clamp M, Barton GJ. 2009. Jalview Version 2: a

670 multiple-sequence alignment editor and analysis workbench. Bioinformatics 25, 1189-1191.

671 Wendel JF, Cronn RC. 2003. Polyploidy and the evolutionary history of cotton. Advances in

672 Agronomy 78, 139-186.

673 Yamaguchi-Shinozaki K, Shinozaki K. 2006. Transcriptional regulatory networks in cellular 674 responses and tolerance to dehydration and cold stresses. Annual Review of Plant Biology 57, 781-803.

675 Yoshida T, Fujita Y, Sayama H, Kidokoro S, Maruyama K, Mizoi J, Shinozaki K, Yamaguchi-

676 Shinozaki, K. 2010. AREB1, AREB2, and ABF3 are master transcription factors that cooperatively

677 regulate $\mathrm{ABRE}$-dependent $\mathrm{ABA}$ signaling involved in drought stress tolerance and require $\mathrm{AB} A$ for full

678 activation. The Plant Journal 61, 672-685.

679 Yoshida T, Mogami J, Yamaguchi-Shinozaki, K. 2014. ABA-dependent and ABA-independent 680 signaling in response to osmotic stress in plants. Current Opinion in Plant Biology 21, 133-139.

681 Yoshida T, Fujita Y, Maruyama K, Mogami J, Todaka D, Shinozaki K, Yamaguchi-Shinozaki K.

682 .2015. Four Arabidopsis AREB/ABF transcription factors function predominantly in gene expression

683 downstream of SnRK2 kinases in abscisic acid signaling in response to osmotic stress. Plant, Cell \&

684 Environment 38, 35-49.

685 Zhou MQ, Shen C, Wu LH, Tang KX, Lin J. 2011. CBF-dependent signaling pathway: a key 686 responder to low temperature stress in plants. Critical Reviews in Biotechnology 31, 186-192. 
688 Table 1. Transcript copy number per ng total RNA and relative expression of selected GhABF

689 expressing transgenic Arabidopsis lines used for phenotypic and abiotic stress tolerance evaluation.

690 Lines selected represent a relatively low level of ectopic expression, the highest level of ectopic

691 expression of the lines quantified, and an approximate average expression level of the low and high

692 expressing lines. Data are means of three biological replicates and three technical replicates \pm SD.

\begin{tabular}{|c|c|c|c|}
\hline Gh homolog & Selected line \# & Transcripts / ng total RNA & Relative expression \\
\hline \multirow[t]{3}{*}{$A B F 1 A$} & 4 & $6.5 \pm 1.21$ & $1.0 \pm 0.24$ \\
\hline & 3 & $144.7 \pm 25.44$ & $16.4 \pm 0.27$ \\
\hline & 6 & $220.1 \pm 52.01$ & $23.9 \pm 0.31$ \\
\hline \multirow[t]{3}{*}{$A B F 1 D$} & 6 & $19.6 \pm 1.39$ & $1.0 \pm 0.11$ \\
\hline & 2 & $121.0 \pm 37.01$ & $5.7 \pm 0.41$ \\
\hline & 5 & $309.7 \pm 58.59$ & $13.9 \pm 0.25$ \\
\hline \multirow[t]{3}{*}{$A B F 2 A$} & 3 & $287.3 \pm 6.29$ & $1.0 \pm 0.03$ \\
\hline & 5 & $372.8 \pm 41.11$ & $1.3 \pm 0.15$ \\
\hline & 9 & $489.7 \pm 41.33$ & $1.7 \pm 0.11$ \\
\hline \multirow[t]{3}{*}{$A B F 2 D$} & 9 & $26.6 \pm 5.88$ & $1.0 \pm 0.31$ \\
\hline & 11 & $177.5 \pm 37.25$ & $7.1 \pm 0.32$ \\
\hline & 25 & $419.5 \pm 114.46$ & $17.3 \pm 0.41$ \\
\hline \multirow[t]{3}{*}{$A B F 3 A$} & 3 & $175.1 \pm 43.54$ & $1.0 \pm 0.39$ \\
\hline & 13 & $1814.5 \pm 358.18$ & $12.3 \pm 0.30$ \\
\hline & 8 & $6383.2 \pm 877.40$ & $47.5 \pm 0.21$ \\
\hline \multirow[t]{3}{*}{$A B F 3 D$} & 1 & $17.2 \pm 3.22$ & $1.0 \pm 0.09$ \\
\hline & 14 & $406.8 \pm 60.34$ & $26.2 \pm 0.21$ \\
\hline & 13 & $770.7 \pm 49.15$ & $50.7 \pm 0.95$ \\
\hline \multirow[t]{3}{*}{$A B F 4 A$} & 2 & $224.5 \pm 14.40$ & $1.0 \pm 0.10$ \\
\hline & 5 & $887.8 \pm 42.06$ & $4.4 \pm 0.08$ \\
\hline & 7 & $1563.3 \pm 190.62$ & $8.1 \pm 0.19$ \\
\hline \multirow[t]{3}{*}{$A B F 4 D$} & 1 & $21.7 \pm 1.47$ & $1.0 \pm 0.09$ \\
\hline & 3 & $42.4 \pm 9.07$ & $1.8 \pm 0.31$ \\
\hline & 7 & $62.5 \pm 13.17$ & $2.7 \pm 0.28$ \\
\hline
\end{tabular}


695 Figure legends

696 Fig. 1. The $A t A R E B / A B F s$ are differentially expressed in response to exogenous ABA, dehydration, and

697 chilling temperatures. Transcript copy number per ng total RNA and relative expression in three week

698 old plants in response to (A-B) $100 \mu \mathrm{M}$ exogenous ABA application, (C-D) dehydration, and (E-F)

699 chilling temperatures $\left(4^{\circ} \mathrm{C}\right)$. Data are means of three biological replicates and three technical replicates $700 \pm$ SD.

702 Fig. 2. The $G h A B F$ homologs are differentially expressed in response to exogenous ABA, dehydration, 703 and chilling temperatures. Transcript copy number per ng total RNA in six to eight week old plants in 704 response to (A-D) $500 \mu \mathrm{M}$ exogenous ABA application, (E-H) dehydration, and (I-L) chilling

705 temperatures $\left(4^{\circ} \mathrm{C}\right)$. Data are means of three biological replicates and three technical replicates \pm SD.

707 Fig. 3. Ectopic GhABF protein expression is largely independent of transcript level. Protein

708 accumulation in eight-day-old seedlings from transgenic lines, compared to WT, expressing

709 GhABF2D, GhABF3D, and GhABF4D treated without and with $50 \mu \mathrm{M}$ ABA for 6 h. Comassie blue

710 staining was used as the loading control (5\% of IP input).

712 Fig. 4. Ectopic expression of the GhABF homologs in Arabidopsis can delay the reproductive

713 transition. (A) Representative images of G. hirsutum ABF expressing transgenic Arabidopsis lines 714 alongside WT Arabidopsis; $\triangle 5$ days. (B) Comparison of the reproductive transition of $G h A B F$ ectopic 715 expressing Arabidopsis lines relative to WT Arabidopsis. Negative values represent a precocious

716 transition, positive values indicate a delay. Data are means of three independent replicates with an 717 average of five plants each $\pm \mathrm{SD}$. Student's $t$-test; $* P<0.05$, ** $P<0.01$.

719 Fig. 5. Ectopic GhABF expression in Arabidopsis can improve tolerance to water deficit and osmotic 720 stress. (A) Relative survival (\%) of transgenic lines as compared to WT Arabidopsis after $6 \mathrm{~h}$

721 dehydration. Data are means of three independent experiments with an average of ten plants each \pm SD.

722 (B) Electrolyte leakage in response to increasingly negative water potentials. Data are means of three

723 independent experiments with three replications each $\pm \mathrm{SD}$. Student's $t$-test; $* P<0.05, * * P<0.01$. 
726 Fig. 6. Ectopic GhABF expression in Arabidopsis can improve cold tolerance, in a gene dependent

727 manner. (A) Relative survival (\%) of transgenic lines as compared to WT Arabidopsis after $5 \mathrm{~h}$ at -7

$728{ }^{\circ} \mathrm{C}$. Data are means of three independent experiments with an average of ten plants each \pm SD. (B)

729 Electrolyte leakage in response to increasingly negative temperatures. Data are means of three

730 independent experiments with three replications each \pm SD. Student's $t$-test; $* P<0.05, * * P<0.01$. 
bioRxiv preprint doi: https://doi.org/10.1101/186015; this version posted September 7, 2017. The copyright holder for this preprint (which was not certified by peer review) is the author/funder, who has granted bioRxiv a license to display the preprint in perpetuity. It is made available under aCC-BY-NC-ND 4.0 International license.

732

\section{Figures}
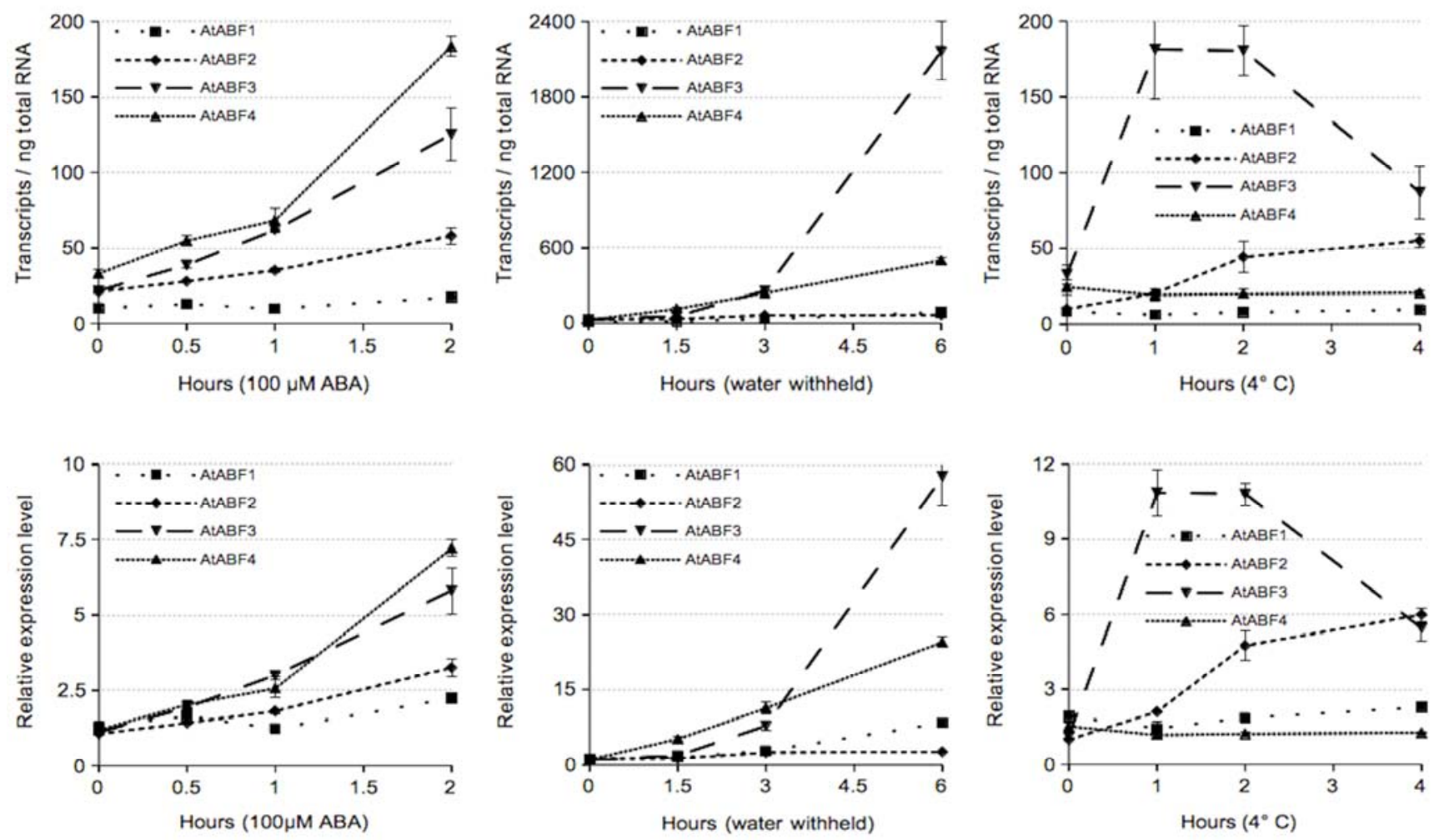

734

735

736

737

738

739 Fig. 1 

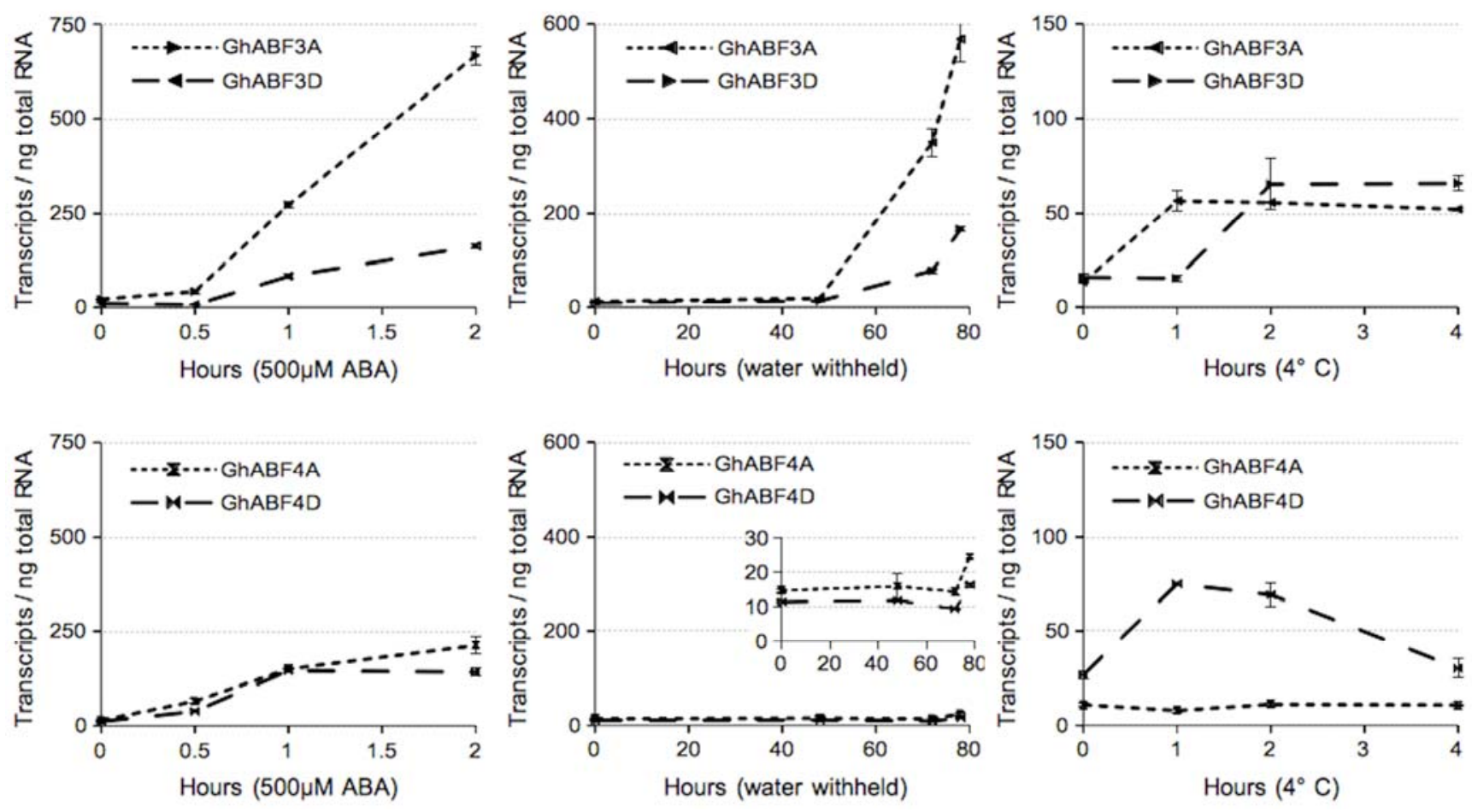

\section{$745 \quad$ Fig. 2}


bioRxiv preprint doi: https://doi.org/10.1101/186015; this version posted September 7, 2017. The copyright holder for this preprint (which was not certified by peer review) is the author/funder, who has granted bioRxiv a license to display the preprint in perpetuity. It is made available under aCC-BY-NC-ND 4.0 International license.

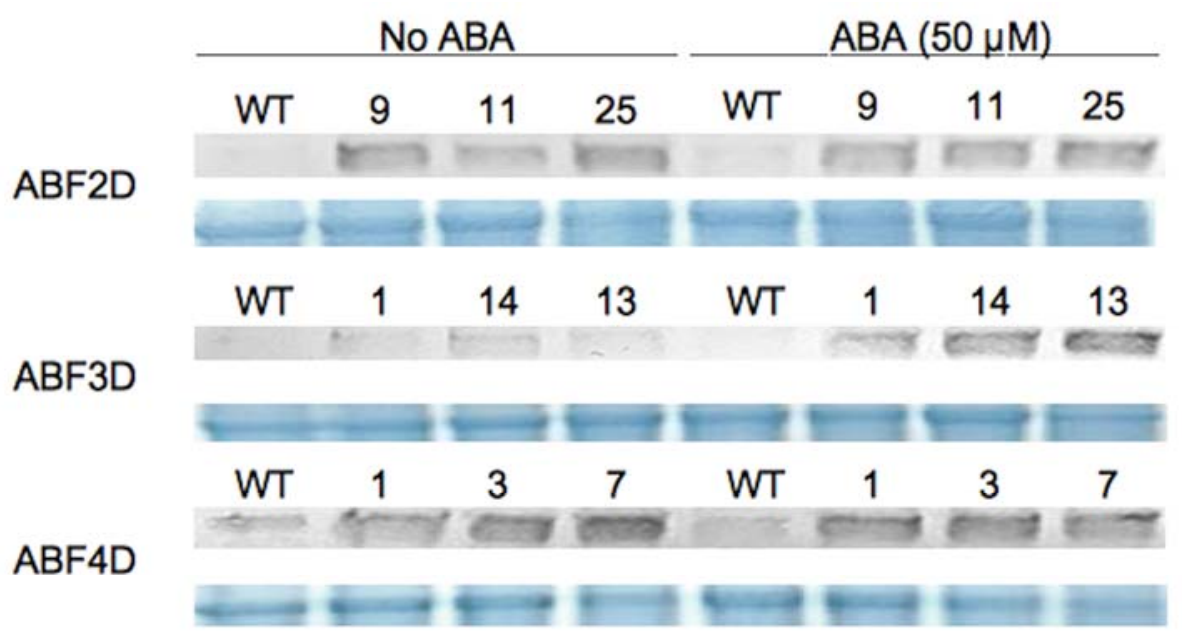

752 Fig. 3 


\section{A}

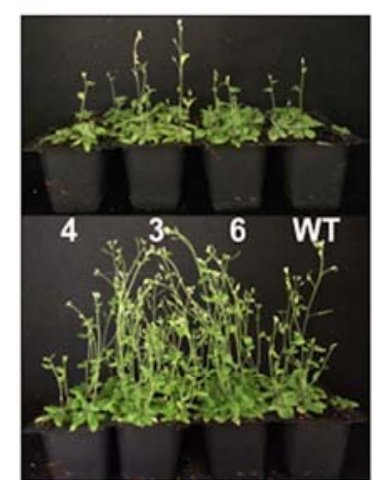

GhABF1A

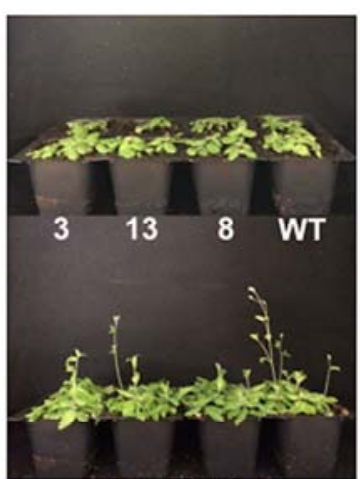

GhABF3A

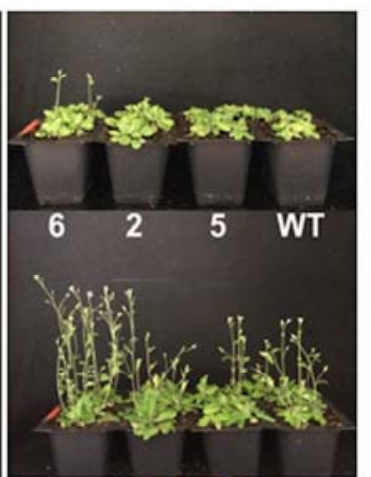

GhABF1D

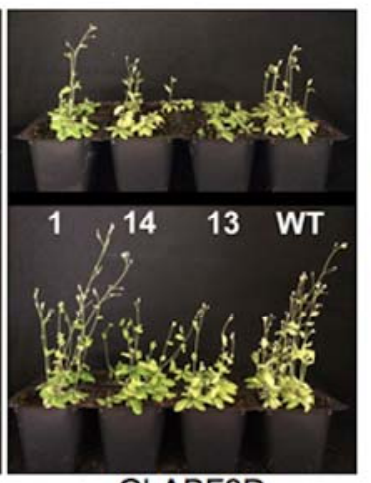

GhABF3D

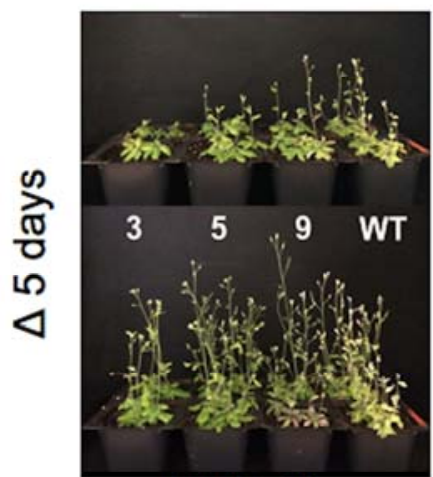

GhABF2A

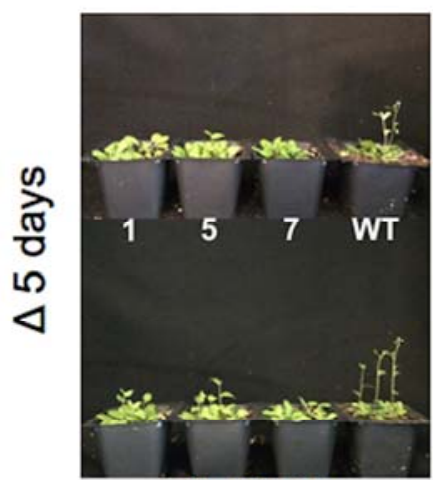

GhABF4A

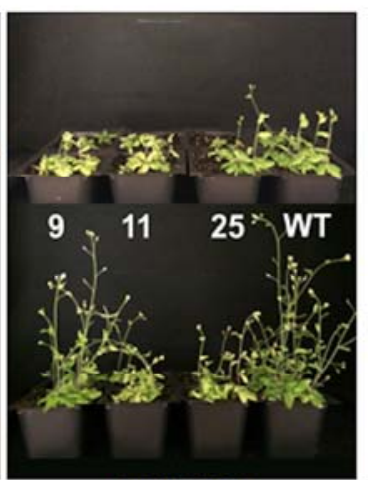

GhABF2D

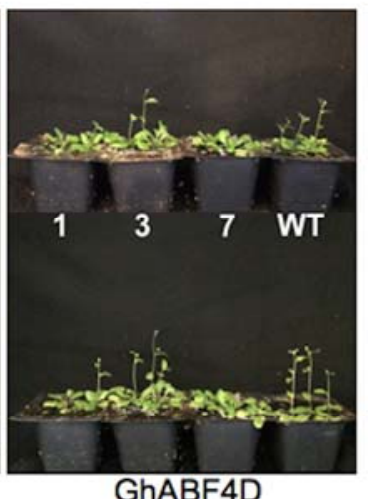

GhABF4D

B

GhABF1A GhABF1D GhABF2A GhABF2D GhABF3A GhABF3D GhABF4A GhABF4D

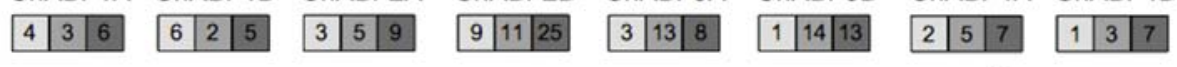

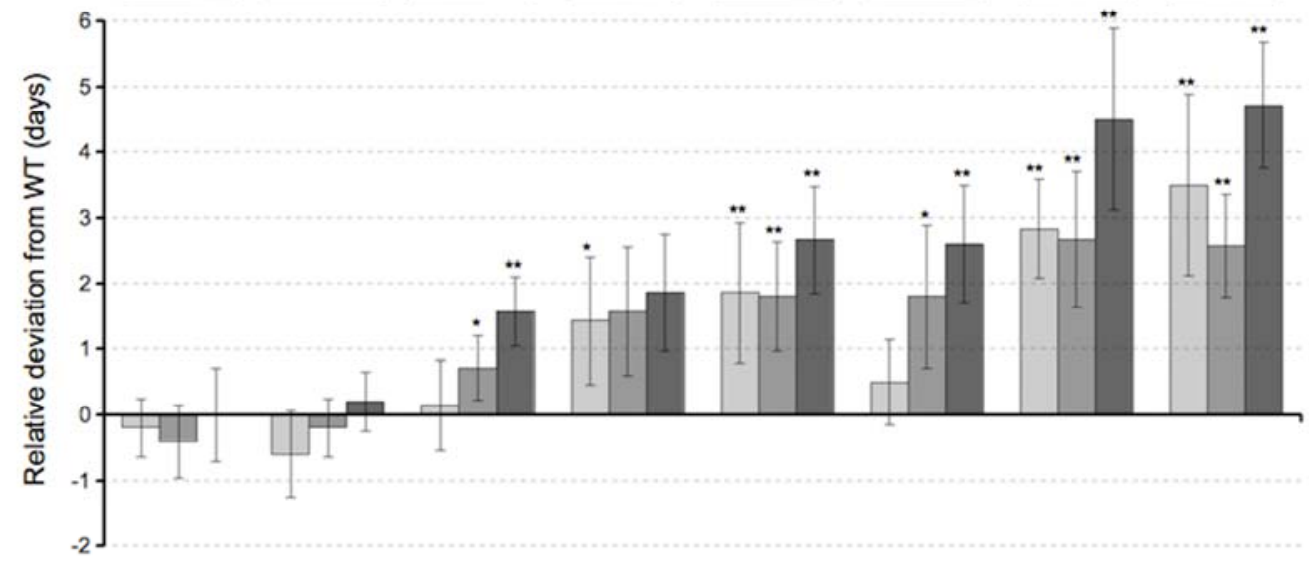

754 Fig. 4 
bioRxiv preprint doi: https://doi.org/10.1101/186015; this version posted September 7, 2017. The copyright holder for this preprint (which was not certified by peer review) is the author/funder, who has granted bioRxiv a license to display the preprint in perpetuity. It is made available under aCC-BY-NC-ND 4.0 International license.

A

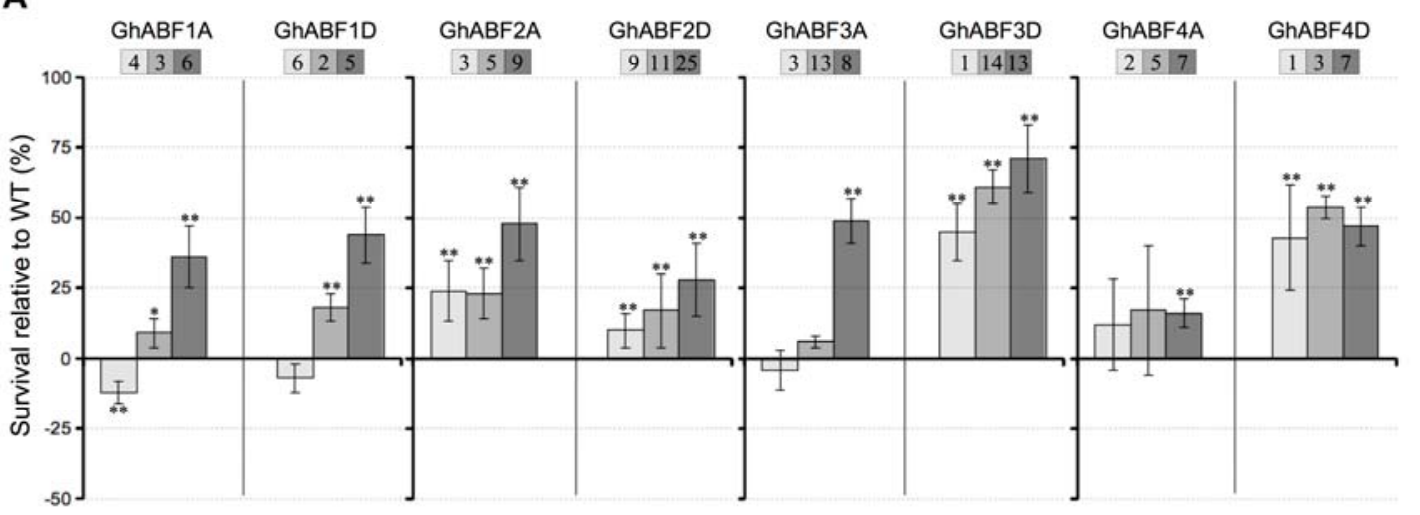

6 hours dehydration

B

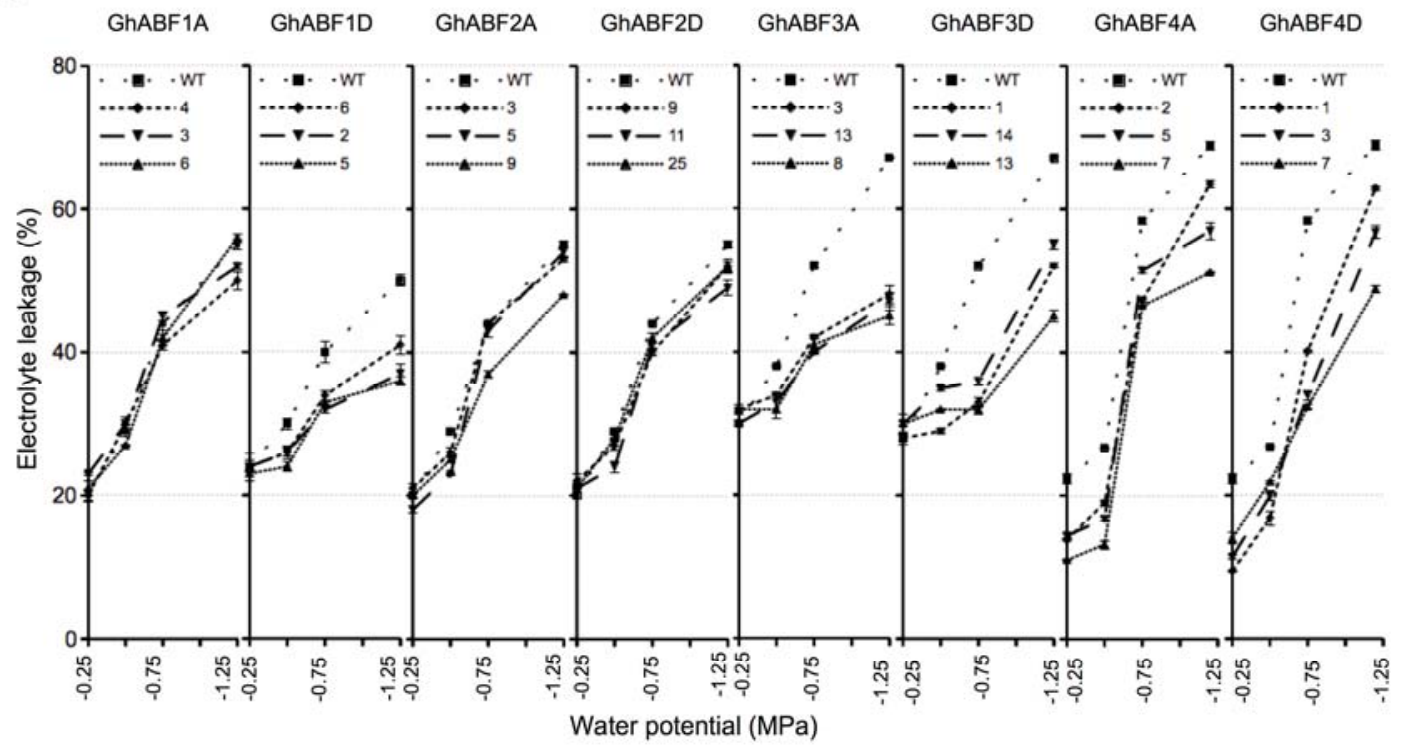

755 Fig. 5 
bioRxiv preprint doi: https://doi.org/10.1101/186015; this version posted September 7, 2017. The copyright holder for this preprint (which was not certified by peer review) is the author/funder, who has granted bioRxiv a license to display the preprint in perpetuity. It is made available under aCC-BY-NC-ND 4.0 International license.
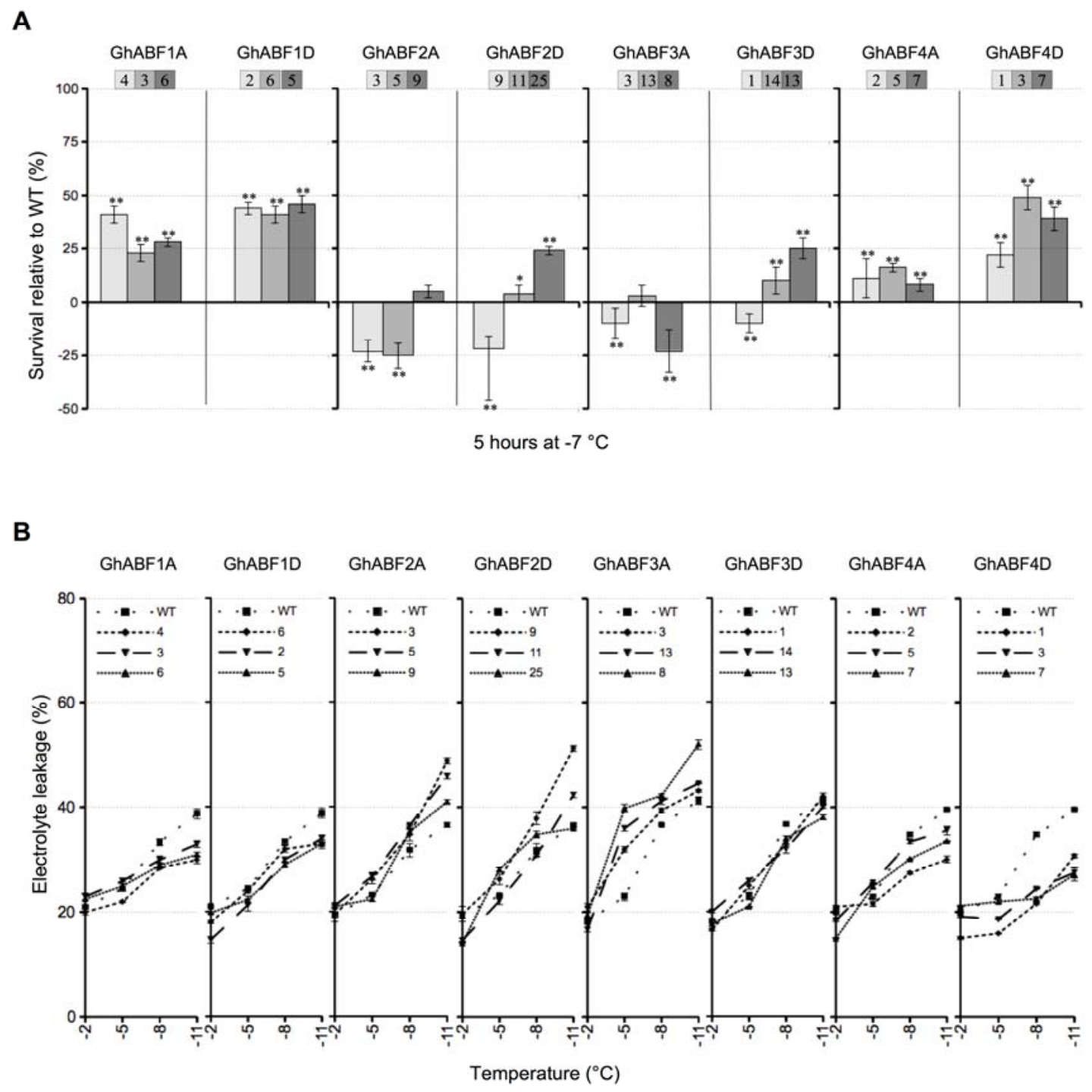

757 Fig. 6 